\title{
GPS availability and positioning issues when the signal paths are aligned with ionospheric plasma bubbles
}

\author{
Alison de O. Moraes ${ }^{1}(1) \cdot$ Bruno C. Vani ${ }^{2} \cdot$ Emanoel Costa $^{3} \cdot$ Mangalathayil A. Abdu $^{4}$ Eurico R. de Paula ${ }^{5}$. \\ Jonas Sousasantos ${ }^{6}$. João F. G. Monico ${ }^{7}$. Biagio Forte ${ }^{8} \cdot$ Patrícia Mara de Siqueira Negreti $^{5}$. \\ Milton Hirokazu Shimabukuro ${ }^{7}$
}

Received: 21 September 2017 / Accepted: 6 July 2018 / Published online: 11 July 2018

(c) Springer-Verlag GmbH Germany, part of Springer Nature 2018

\begin{abstract}
The propagation paths of signals through equatorial ionospheric irregularities are analyzed by evaluating their effects on Global Navigation Satellite System (GNSS) positioning and availability. Based on observations during 32 days by a scintillation monitor at São José dos Campos, Brazil, it was noted that there is a dominance of enhanced scintillation events for Global Positioning System (GPS) ray paths aligned with the azimuth angle of $345^{\circ}$ (geographic northwest). This azimuth corresponds to the magnetic meridian that has a large westward declination angle in the region $\left(21.4^{\circ} \mathrm{W}\right)$. Such results suggest that the enhanced scintillation events were associated with GPS signals that propagated through plasma bubbles aligned along the direction of the magnetic field. It will be shown that, under this alignment condition, the longer propagation path length through plasma bubbles can result in more severe scintillation cases and more losses of signal lock, as supported by proposed statistics of bit error probability and mean time between cycle slips. Additionally, large precise positioning errors are also related to these events, as demonstrated by precise point positioning experiments.
\end{abstract}

Keywords Ionospheric scintillation · Fading distribution · GPS availability

Alison de O. Moraes

alisonaom@iae.cta.br

Bruno C. Vani

brunovani@ifsp.edu.br

Emanoel Costa

epoc@cetuc.puc-rio.br

Mangalathayil A. Abdu

ma.abdu@inpe.br

Eurico R. de Paula

eurico.paula@inpe.br

Jonas Sousasantos

jonasjss@ita.br

João F. G. Monico

galera@fct.unesp.br

Biagio Forte

B.Forte@bath.ac.uk

Patrícia Mara de Siqueira Negreti

patricia.mara@dae.inpe.br

Milton Hirokazu Shimabukuro

miltonhs@fct.unesp.br
1 Instituto de Aeronáutica e Espaço, IAE/Instituto Tecnológico de Aeronáutica, ITA, São José dos Campos, SP, Brazil

2 Instituto Federal de Educação, Ciência e Tecnologia de São Paulo, Campus Presidente Epitácio (IFSP-PEP), Presidente Epitácio, SP, Brazil

3 Centro de Estudos em Telecomunicações, Pontifícia Universidade Católica do Rio de Janeiro (CETUC/PUC-Rio), Rua Marquês de São Vicente 225, Rio de Janeiro, RJ 22451-900, Brazil

4 Instituto Tecnológico de Aeronáutica, ITA/Instituto Nacional de Pesquisas Espaciais, INPE, São José dos Campos, SP, Brazil

5 Instituto Nacional de Pesquisas Espaciais, INPE, São José dos Campos, SP, Brazil

6 Instituto Tecnológico de Aeronáutica, ITA, São José dos Campos, Brazil

7 Universidade Estadual Paulista Júlio de Mesquita Filho, UNESP, Presidente Prudente, SP, Brazil

8 University of Bath, Bath, UK 


\section{Introduction}

Ionospheric scintillation degrades the accuracy of navigation and positioning based on Global Navigation Satellite Systems (GNSS). Some authors observed that rapid phase variations are mapped into Doppler shifts in the Global Positioning System (GPS) signal, which may exceed the bandwidth of the phase lock loop, resulting in a loss of phase lock (Doherty et al. 2004). Additionally, amplitude fading may cause the signal-to-noise ratio to drop below the receiver threshold. These effects often accompany propagation delays and increase range measurement errors, besides causing the carrier and code loops to lose lock (Kintner et al. 2001). Scintillation may seriously affect positioning, resulting in total disruption of the receiver operation (Basu and Basu 1981). Such extreme phenomena are more usual near the equatorial and lowlatitude regions between $\pm 20^{\circ}$ geomagnetic latitude than in the auroral and polar zones above $55^{\circ}$ latitude. This work analyzes GPS scintillation data recorded during 32 days of the current solar maximum conditions at São José dos Campos, Brazil, whose geographic coordinates are $23.2^{\circ} \mathrm{S}, 45.9^{\circ} \mathrm{W}$, and dip latitude: $19.2^{\circ} \mathrm{S}$, near the southern crest of the equatorial ionization anomaly (EIA). The analysis has been performed by relating the scintillation intensity with the geometry of the propagation paths through the ionospheric irregularities and then evaluating the effects on GPS positioning and availability. The nature of the plasma bubble irregularity distribution with respect to the geomagnetic field configuration may magnify the scintillation effects. In Brazil, the geomagnetic field configuration, characterized by a large magnetic declination angle, provides a particular and favorable condition to assess such effects. A recent study by Moraes et al. (2017) showed scintillation enhancement events around the azimuth angle of $345^{\circ}$, which corresponds to signal propagation along ray paths that are nearly aligned with the magnetic meridian in this region. In view of the large westward magnetic declination $\left(21.4^{\circ} \mathrm{W}\right)$ of the region, this result suggested that the enhanced scintillation events were associated with GPS signals that propagated along plasma bubbles aligned along the direction of the magnetic field. Calculations of the propagation angles with respect to the magnetic field lines showed that small values of this parameter and the larger propagation path length through bubbles could result in severe scintillation and more cases of loss of phase lock. This analysis will also show that large errors on precise point positioning (PPP) are related to the eventual alignment.

Recently, Humphreys et al. (2009, 2010a) have suggested that deep fades in the amplitude of received signals, simultaneously occurring with sudden phase changes, caused loss of phase lock in carrier tracking loops of GPS receivers. Such occurrences, also known as "canonical fades", have been observed during strong low-latitude scintillation events (Humphreys et al. 2010b). In the present work, the canonical fading will be examined as a likely consequence of GPS signal propagation paths being aligned with the plasma bubble. These nonlinear ionospheric propagation effects on the GPS radio signal will be analyzed using the fading coefficients of the $\alpha-\mu$ distribution (Yacoub 2007).

The next section will survey ionospheric scintillation. After a brief description of the experimental setup, the period of analysis and the geophysical condition will be presented. We will then present a formulation that estimates the mean time between cycle slips in the receiver tracking loop based on its observed relation with the bit error probability (Humphreys et al. 2010b). Next, the relation between scintillation parameters and propagation path directions will be analyzed, and evidence of the effects of this irregularity alignment on the GPS PPP will be presented. The final section summarizes the main results of the study and presents concluding remarks.

\section{Scintillation-producing ionospheric irregularities}

Plasma irregularities may develop in the post-sunset equatorial ionosphere under the electrodynamic processes unique to the sunset transition. The pre-reversal enhancement in the evening zonal electric field causes rapid uplifts of the steep gradient region of the bottom side ionospheric $\mathrm{F}$ layer. This establishes the conditions for the interchange instability processes driven by the Rayleigh-Taylor (R-T) mechanism to act on density perturbations, which begin to grow. The nonlinear growth of the irregularities involves the bottomside lower density plasma rising up even further to the topside ionosphere, in the form of magnetic flux tube aligned plasma depletions known as equatorial plasma bubbles, with extremities extending to latitudes of larger background plasma density in the EIA. During their evolution, the EPBs are observed to drift generally eastward. Motions of medium-scale density secondary structures across transionospheric paths form complex moving field patterns at the ground that contain amplitude and phase variations. In this way, random temporal fluctuations are produced in both amplitude and phase of satellite signals received at the ground, which are known as amplitude and phase scintillations, respectively (Yeh and Liu 1982). The irregularities with scale sizes of a few hundred meters are responsible for producing scintillation of transionospheric signals emitted by GNSS satellites (Kintner et al. 2007). Under geomagnetically quiet conditions, the equatorial scintillation activity develops in the post-sunset hours and typically lasts for 4-5 h until midnight, sometimes also extending for a few 
hours into the post-midnight period. It presents strong seasonal and longitudinal variations that are dependent on the corresponding variations in the alignment between the sunset terminator and the magnetic meridian, as well as on the availability of seeding sources (Abdu et al. 1981; Tsunoda 1985). In the Brazilian region, scintillation activity extends from September to March and peaks around December. In addition, short-term variability may occur due to upward propagating atmospheric waves, sudden stratospheric warming episodes, and changes in solar and magnetic activities (de Paula et al. 2015; Abdu et al. 2015).

EPBs have an east-west extension of a few tens to hundreds of kilometers and often occur in succession with zonal separation of a few hundred kilometers. Such features may be noted as the magnetic north-south-aligned depletion patches in the total electron content distribution map constructed from measurements using GPS receiver arrays, whose units are $10^{16} \mathrm{el} / \mathrm{m}^{2}$. Some examples of such TEC maps over Brazil, using data from November 17-28, 2014, are shown in Fig. 1. Note that the TEC depletions, associated with equatorial plasma bubbles, extend from the EIA trough region, along the dip equator, to the EIA crest region of larger background TEC values. We also note a large degree of day-to-day variability in the TEC depletions, as well as in the background TEC values. Two situations lead to intense scintillation. The first situation is the larger background plasma density that exists in the crest region of the EIA, where the plasma irregularities are more intense. The second situation, which is the focus of the present study, is the degree to which segments of signal propagation paths become closer to being aligned with the field-aligned plasma bubble structures. Since scintillation results from effects integrated along the propagation path, the longer the field-aligned propagation segment, the more intense will the amplitude and the phase scintillation be. In other words, the geomagnetic declination and inclination angles, associated with the GPS signal paths of the ionospheric pierce point (IPP) distribution, are controlling factors of the scintillation distribution surrounding a receiving site.

\section{Ionospheric scintillation measurements, spatial distribution and alignment}

The scintillation data used in the present study were recorded by a Septentrio PolaRxS monitor belonging to the CIGALA/CALIBRA network (Vani et al. 2016). This monitor is located at São José dos Campos (SJC), Brazil, whose geographic coordinates are: $23.2^{\circ} \mathrm{S}, 45.9^{\circ} \mathrm{W}$, dip latitude: $19.2^{\circ} \mathrm{S}$. The analysis focuses on two periods: November 15-30, 2014 and February 4-18, 2015. The average sunspot number and the F10.7 solar flux values varied around 169 and 133 s.f.u., respectively, where 1 s.f.u. $=10^{-22} \mathrm{~W} / \mathrm{m}^{2} / \mathrm{Hz}$. The measurements were made during the equatorial spread
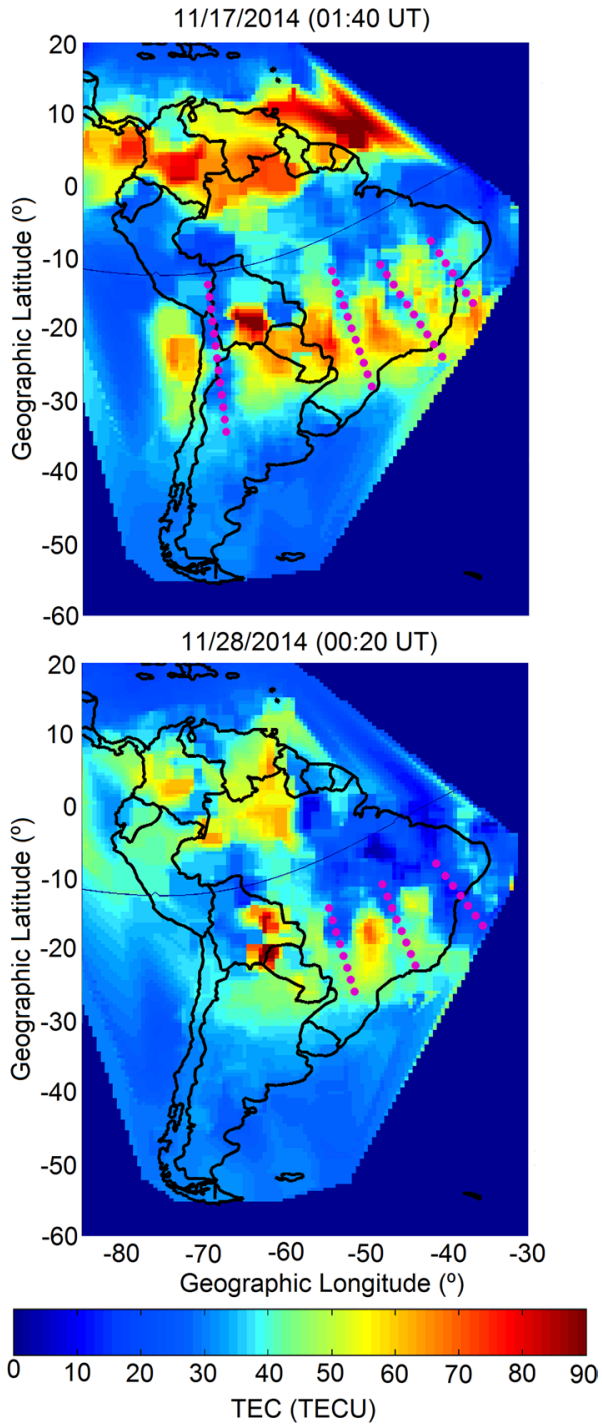

Fig. 1 Examples of TEC maps over Brazil, showing the magnetic north-south-aligned bubble structures (pink dots) with extremities extending several degrees to lower geomagnetic latitudes. In addition, shown are, in red and brown, the larger background TEC values in the EIA crest region

F occurrence season in Brazil, which typically extends from September to April (Sobral et al. 2002). For this study, GPS satellites with elevations greater than $20^{\circ}$ were considered. In the set of observations from 19:00 LT to 01:00 LT during 32 nights, approximately $179 \mathrm{~h}$ of significant GPS L1 amplitude scintillation were recorded, considering the transmissions from all satellites.

The strength of the amplitude scintillation, represented by the $\mathrm{S}_{4}$ index, defined as the normalized standard deviation of the received signal intensity (Yeh and Liu 1982),

$S_{4}=\sqrt{\left\langle I^{2}\right\rangle-\langle I\rangle^{2}} /\langle I\rangle$, 
is one of the basic parameters for this study. In this wellknown expression, $I=|R|^{2}$ is the received intensity signal, $R$ is its amplitude, and $<>$ denote an ensemble average during $1 \mathrm{~min}$. The receiver records the intensity at $50 \mathrm{~Hz}$ for all tracked satellites, providing the respective $S_{4}$ index at every $60 \mathrm{~s}$. Only samples with $S_{4}>0.3$ were considered as scintillation cases. The first Fresnel zone scale sizes of the fieldaligned irregularities that are responsible for scintillation, immersed in the larger-scale plasma bubbles, are approximately equal to $400 \mathrm{~m}$ at the GPS L-band frequencies.

Figure 2 shows the azimuth and elevation distributions of amplitude scintillation observed by the São José dos Campos monitor during the two periods of observations. All 10,743
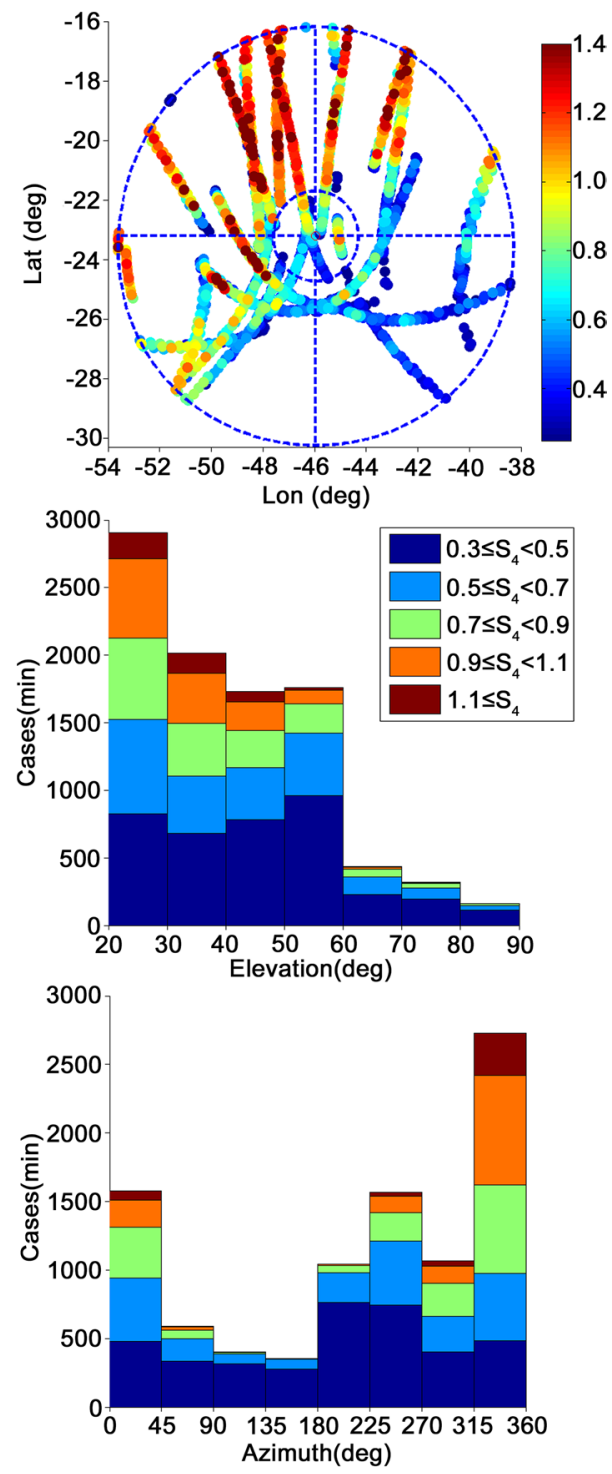

Fig. 2 GPS L1 amplitude scintillation events plotted at the respective IPP values (top panel). The outer and inner circles define elevations of $20^{\circ}$ and $60^{\circ}$. The middle and bottom panels show $S_{4}$ distributions as functions of elevation and azimuth, respectively scintillation cases with $S_{4}>0.3$ were used in this plot. The top panel of Fig. 2 shows the azimuth-elevation distribution of the $S_{4}$ values in association with the $350 \mathrm{~km}$ IPPs, using the color scale on the right to represent the scintillation strength. Note that consecutive orbits show very small day-to-day variations during the period of observation. To emphasize the severity of scintillation effects on GPS transmissions, the highest $S_{4}$ values were brought to the front in this plot, showing the predominance of moderate-to-strong amplitude scintillation levels with $S_{4}>0.9$ in the northern sector. This feature may be partly due to the ambient plasma density since TEC must be stronger in the northern sector over São José dos Campos, which includes the crest of the EIA. Scintillation is highly dependent on the apex altitude of the irregularities. The apex altitudes, corresponding to the aligned cases in the northern sector, vary from 431 to $556 \mathrm{~km}$. Equatorial plasma bubbles commonly reach these apex altitudes over Brazil (Abdu et al. 2009). In principle, bubble irregularities may also extend to regions southward of the receiving site. However, only weak signatures of such bubble irregularities were observed in the available data set.

The middle and bottom bar charts in Fig. 2 represent the elevation and azimuth distributions of scintillation occurrences for $\mathrm{S}_{4}$ values varying from $0.3 \leq S_{4}<0.5$ to $S_{4}>1.1$. Note that $80 \%$ of the cases with $S_{4} \geq 1.1$ are associated with elevations less than $41^{\circ}$. For $0.9 \leq S_{4}<1.1$ and $0.7 \leq S_{4}<0.9$ and the same elevation interval, the corresponding percentages are 75 and $73 \%$, respectively. The azimuth chart confirms in quantitative terms the predominance of high $\mathrm{S}_{4}$ values between azimuths $315^{\circ}$ and $360^{\circ}$. For this azimuth sector, $S_{4} \geq 1.1,0.9 \leq S_{4}<1.1$, and $0.7 \leq S_{4}<0.9$ make up 68, 62 , and $40 \%$ of the observed data, respectively. These results are consistent with the ones provided by Xu et al. (2012).

\section{Cycle slips, bit error rate and $a-\mu$ model}

Humphreys et al. (2010b) showed that the bit error probability $P_{\mathrm{e}}$ for binary differential phase-shift keying (binary DPSK) transmissions and the mean time between cycle slips $T_{\mathrm{s}}$ are closely related by a $T_{\mathrm{s}} \approx T_{\mathrm{b}} / P_{\mathrm{e}}$, where $T_{\mathrm{b}}=0.02 \mathrm{~s}$ is the GPS L1 bit duration. Based on this connection, and assuming a Ricean fading channel, they proposed a model for estimating the cycle slip rate as a function of scintillation intensity, the fluctuation speed, and the carrier power to noise power density ratio.

Based on the work of Yacoub (2007), Moraes et al. (2012, 2014a) used the $\alpha-\mu$ model to provide a flexible and realistic description of the amplitude scintillation. The $\alpha-\mu$ probability density function (pdf) of the amplitude envelope $R$ of the received signal is given by,

$f_{\mathrm{R}}(r)=\frac{\alpha \mu^{\mu}}{\Gamma(\mu) \tilde{r}}(r / \tilde{r})^{\alpha \mu-1} \exp \left[-\mu(r / \tilde{r})^{\alpha}\right]$, 
where $\alpha>0$ is an arbitrary fading parameter, $\tilde{r}=\left[E\left(R^{\alpha}\right)\right]^{1 / \alpha}$, and $\mu>0$ is the inverse of the normalized variance of $R^{\alpha}$; that is, $\mu=E^{2}\left(R^{\alpha}\right) /\left\{E\left(R^{2 \alpha}\right)-E^{2}\left(R^{\alpha}\right)\right\}$. Additionally, $\Gamma(z)$ is the Gamma function of the argument $z$.

Based on the evidence provided by Humphreys et al. (2010b) and considering binary DPSK transmissions, $P_{\mathrm{e}}$, and consequently $T_{\mathrm{s}}$, will be estimated using the $\alpha-\mu$ model. To compute $P_{\mathrm{e}}$, the $\alpha-\mu$ probability density function of the instantaneous signal-to-noise ratio $S=\tilde{s}(R / \tilde{r})^{2}$, where $\tilde{s}=\tilde{r}^{2}\left(E_{\mathrm{b}} / N_{\mathrm{o}}\right)$, and $\left(E_{\mathrm{b}} / N_{\mathrm{o}}\right)$ is the energy per bit to noise power density ratio, is initially obtained from (2) by a straightforward derivation (Magableh and Matalgah 2009)

$f_{\mathrm{S}}(s)=\frac{\alpha \mu^{\mu}}{2 \Gamma(\mu) \tilde{s}}(s / \tilde{s})^{\alpha \mu / 2-1} \exp \left[-\mu(s / \tilde{s})^{\alpha / 2}\right]$.

Note that $\left(E_{\mathrm{b}} / N_{\mathrm{o}}\right)=T_{\mathrm{b}}\left(C / N_{\mathrm{o}}\right)$. Considering $E\left(R^{2}\right)=1$, one gets,

$$
\begin{aligned}
\tilde{r}^{2} & =\frac{\mu^{2 / \alpha} \Gamma(\mu)}{\Gamma(\mu+2 / \alpha)} \rightarrow \tilde{s}=\frac{\mu^{2 / \alpha} \Gamma(\mu)}{\Gamma(\mu+2 / \alpha)}\left(E_{\mathrm{b}} / N_{\mathrm{o}}\right) \\
& =\left[\frac{\mu^{2 / \alpha} \Gamma(\mu)}{\Gamma(\mu+2 / \alpha)}\right] T_{\mathrm{b}}\left(C / N_{\mathrm{o}}\right) .
\end{aligned}
$$

For binary DPSK, $P_{\mathrm{e}}$ is given by (El Ayadi and Ismail 2014)
$P_{\mathrm{e}}=\int_{0}^{\infty} \frac{1}{2} \exp (-s) f_{\mathrm{S}}(s) \mathrm{d} s$

Combining (3)-(5) and changing the integration variable, $P_{\mathrm{e}}$ can be computed by,

$P_{\mathrm{e}}=\frac{\alpha \mu^{\mu}}{4 \Gamma(\mu)} \int_{0}^{\infty} v^{\alpha \mu / 2-1} \mathrm{e}^{-\left(\mu v^{\alpha / 2}+\tilde{s} v\right)} \mathrm{d} v$.

Several formulations for $P_{\mathrm{e}}$ calculation have generally adopted the moment generating function (MGF) approach to express their final results in closed forms, in terms of transcendental functions (Magableh and Matalgah 2009; El Ayadi and Ismail 2014). However, (6) may be easily and accurately calculated by numerical quadrature methods.

The left panels of Fig. 3, obtained from (6) through the latter approach, illustrate the dependence of $P_{\mathrm{e}}$ as a function of $C / N_{\mathrm{o}}$, for $S_{4}=1.1$ (upper left), $S_{4}=0.8$ (lower left), and different values of $\alpha, \mu$, and $\tilde{r}$. Using the $\alpha-\mu$ model, it is more intuitive to analyze scintillation based on $S_{4}$, having $\alpha$ as an auxiliary severity indicator. Indeed, Moraes et al. (2014b) established the following relation between the scintillation index $S_{4}$ and the parameters of the $\alpha-\mu$ distribution.
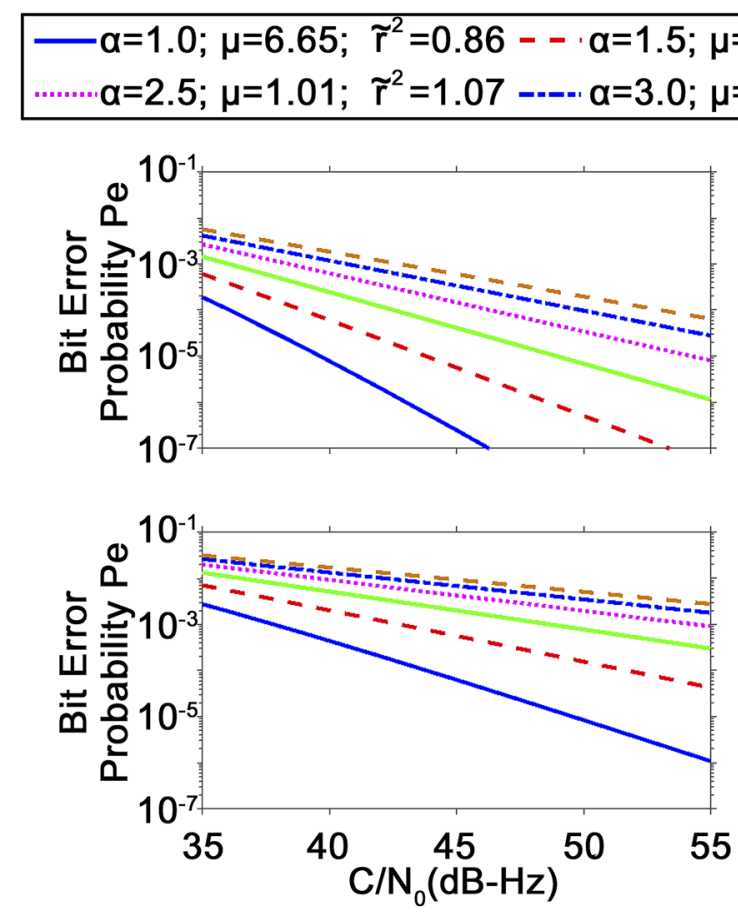

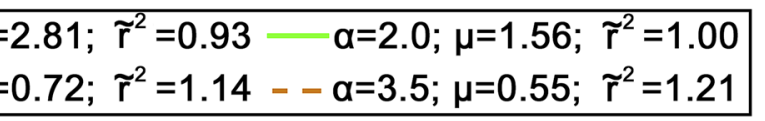
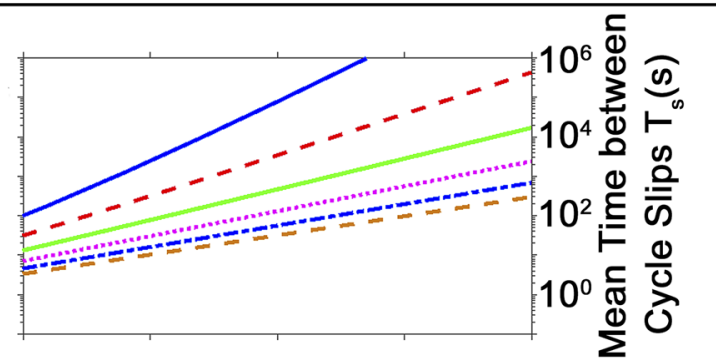

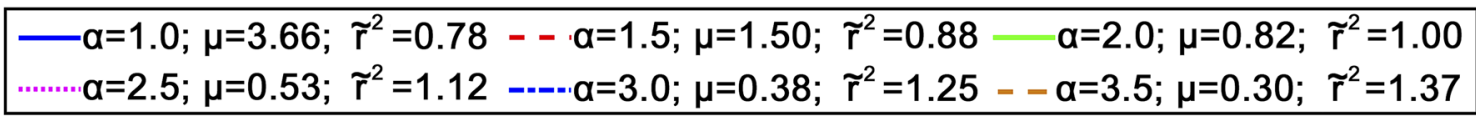

Fig. 3 Curves for $P_{\mathrm{e}}$ (left panels) and $T_{\mathrm{s}}$ (right panels) as functions of $C / N_{\mathrm{o}}$ and different values of $\alpha$ (and associated values of $\mu$ and $\tilde{r}$ ). The upper and bottom panels assume $S_{4}=1.1$ and $S_{4}=0.8$, respectively 
$S_{4}^{2}=\frac{\Gamma(\mu) \Gamma(\mu+4 / \alpha)-\Gamma^{2}(\mu+2 / \alpha)}{\Gamma^{2}(\mu+2 / \alpha)}$.

This equation numerically leads to a $\mu$ value for each pair of independent parameters $S_{4}$ and $\alpha$, and the associated $\tilde{r}$ value is immediately obtained from (4). Equation (7) indicates that the $\alpha-\mu$ model may describe different scintillation patterns for each value of $S_{4}$. This is especially interesting for strong scintillation, since it is well known that $\mathrm{S}_{4}$ alone does not describe the effects from ionospheric perturbations on GPS signals. The left panels of Fig. 3 shows two examples of bit error probability $P_{\mathrm{e}}$, for $S_{4}=0.8$ in the upper panel, and $S_{4}=1.1$ in the lower panel. It is possible to observe that $P_{\mathrm{e}}$ decreases as $\mathrm{C} / \mathrm{N}_{\mathrm{o}}$ increases. The left panels illustrate, for fixed values of $S_{4}$ and different $\alpha$ values, how $P_{\mathrm{e}}$ varies. For example, in case of $C / N_{\mathrm{o}}=42 \mathrm{~dB}-\mathrm{Hz}$ and $S_{4}=1.1$, the estimated values for $P_{\mathrm{e}}$ on the lower-left panel of Fig. 3 are $2.1 \times 10^{-4}, 3.6 \times 10^{-3}$ and $1.4 \times 10^{-2}$, for $\alpha=1.0,2.0$ and 3.5 , respectively. For $S_{4}=0.8$, the corresponding estimates on the upper-left panel of Fig. 3 are $2.0 \times 10^{-6}, 1.0 \times 10^{-4}$, and $1.0 \times 10^{-3}$, for $\alpha=1.0,2.0$, respectively. The legends display, the respective values of $\mu$ and $\tilde{r}$ for these plots.

The right panels of Fig. 3 simply map $P_{\mathrm{e}}$ into the mean time between cycle slips through the approximate relation$\operatorname{ship} T_{\mathrm{s}} \approx T_{\mathrm{b}} / P_{\mathrm{e}}$. Correspondingly, these panels show that $T_{\mathrm{S}}$ decreases as $S_{4}$ increases and each curve shows that $T_{\mathrm{S}}$ increases as $C / N_{\mathrm{o}}$ also increases. Analogously to the $P_{\mathrm{e}}$ curves, in the lower right panel, for $C / N_{\mathrm{o}}=42 \mathrm{~dB}-\mathrm{Hz}$ and $S_{4}=1.1$, the estimated values of $T_{\mathrm{S}}$ are $94.90,5.33$ and $1.48 \mathrm{~s}$, for $\alpha=1.0,2.0$ and 3.5, respectively. In the upperright panel, for $S_{4}=0.8$, the corresponding estimates are $9.53 \times 10^{3}, 161$ and $15.5 \mathrm{~s}$. The above results illustrate how signals with similar $S_{4}$ and $C / N_{\mathrm{o}}$ may have completely different effects on $P_{\mathrm{e}}$ and $T_{\mathrm{s}}$.

The scintillation monitor records $S_{4}$ and average $C / N_{\mathrm{o}}$, but also a flag indicating whether cycle slips occurred during the $1 \mathrm{~min}$ of high-resolution $(50 \mathrm{~Hz})$ measurements. The estimated values of $\alpha, \mu, P_{\mathrm{e}}$ and $T_{\mathrm{s}}$ are also associated with each 1-min record. Figure 4 displays the estimated $T_{\mathrm{s}}$ for the records in which cycle slips occurred, with each symbol representing the corresponding values of $S_{4}, \alpha$, and $T_{\mathrm{s}}$. For a fixed value of $S_{4} \pm 0.025$, each scattered plot relates $T_{\mathrm{S}}$ to $\alpha$, reinforcing that: (1) on average, $T_{\mathrm{s}}$ decreases with increasing values of $S_{4}$; and (2) for a fixed value of $S_{4}$, an increase in $\alpha$ indicates an increase in the number of fades and an associated decrease in $T_{\mathrm{s}}$ (Moraes et al. 2014a). The observed dispersion in $T_{\mathrm{s}}$ for fixed values of $S_{4}$ and $\alpha$ is due to variations in $C / N_{\mathrm{o}}$. It should be remembered that the Septentrio PolaRxS GPS monitor is not a standard configuration. Instead, it is optimized for tracking through periods of scintillation. Thus, the above distribution of cycle slips is very likely different from those of normal GPS receivers in quantitative terms. However, it is expected that the

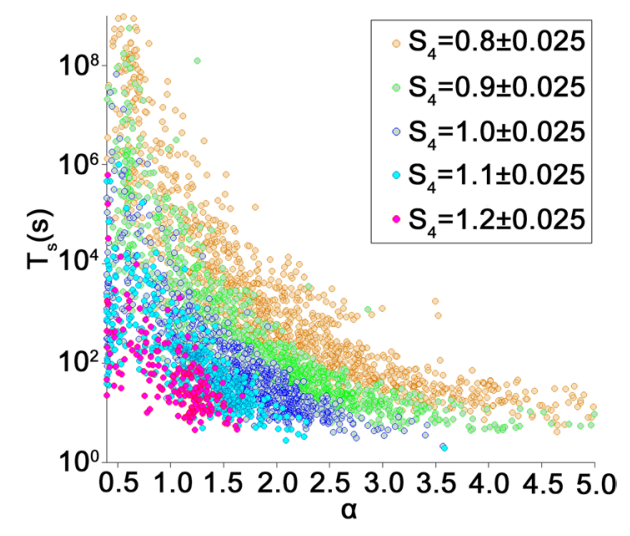

Fig. 4 Cycle slips detected by the scintillation monitor for different $S_{4} \pm 0.025$ ranges

qualitative principles extracted from the above results would also be applicable to them.

\section{Alignment and availability}

This section associates $P_{\mathrm{e}}$ and $T_{\mathrm{s}}$ with the angle between the propagation path and the geomagnetic field directions. To obtain this angle, the propagation path direction lines (in terms of their azimuth and elevation angles) at the $350 \mathrm{~km}$ IPPs were initially calculated for the receiver location and all satellite positions at that moment. The latest version of the International Geomagnetic Reference Field (IGRF-12) (Thébault et al. 2015) provided the geomagnetic field line directions at the IPPs. Next, all determined lines were projected onto the vertical, i.e., north and up, and horizontal, i.e., north and east planes at the IPPs. The first projection $(\omega)$ measures the difference between the path elevation and the geomagnetic inclination. Note that the path elevations at the IPPs displayed by Fig. 2, measured from the horizontal plane toward zenith at the IPP, are always greater than $20^{\circ}$, as explained in the first paragraph of the third section. Similarly, the geomagnetic inclination line is also above the same horizontal plane, since the dip angle is negative in the southern hemisphere. However, the difference $\omega$ can be negative or positive, indicating that the path elevation line is below or above the geomagnetic inclination line, with respect to that horizontal plane, respectively. The second projection $(\nu)$ represents the angle between the path azimuth and the geomagnetic declination lines on the horizontal plane through the IPP. While the path azimuths at the IPPs displayed by Fig. 2 vary from $0^{\circ}$ to $360^{\circ}$, the angle $\nu$ can be negative, indicating that the path azimuth line is to the right of the geomagnetic declination line, or positive, if the other relative position holds. Thus, the set of parameters associated with each 1-min record, listed in the last paragraph of the previous section, is augmented with $\omega$ and $\nu$. The present study assumed that if $|\omega|$ and $|\nu|$ are simultaneously less than $10^{\circ}$, 
the GPS propagation path would be effectively aligned with the ionospheric bubble irregularity structure.

The bar plots in the top and middle panels of Fig. 5 display the number of L1 scintillation occurrences as a function of $\omega$ and $\nu$, for different ranges of $S_{4}$. It is noted that the smallest $\omega$ are related with the largest scintillation index interval, and vice-versa. Similarly, the largest $S_{4}$ values occur when the difference between the propagation angle and the magnetic declination is the smallest. Thus, a small angle between the propagation direction and the field-aligned bubbles, on both vertical and horizontal planes, corresponds to large scintillation intensity. The bottom panel of Fig. 5 shows the percentages of aligned and nonaligned cases, which always add to $100 \%$ within each $S_{4}$ interval. It indicates that the increasing percentage of alignment between the propagation angle and magnetic field line results in a severe increase of the scintillation intensity.

The top panel of Fig. 6 shows the estimated $T_{\mathrm{s}}$ values as functions of $S_{4}$, where the colors red and green represent aligned and nonaligned cases, respectively. It is noticed that
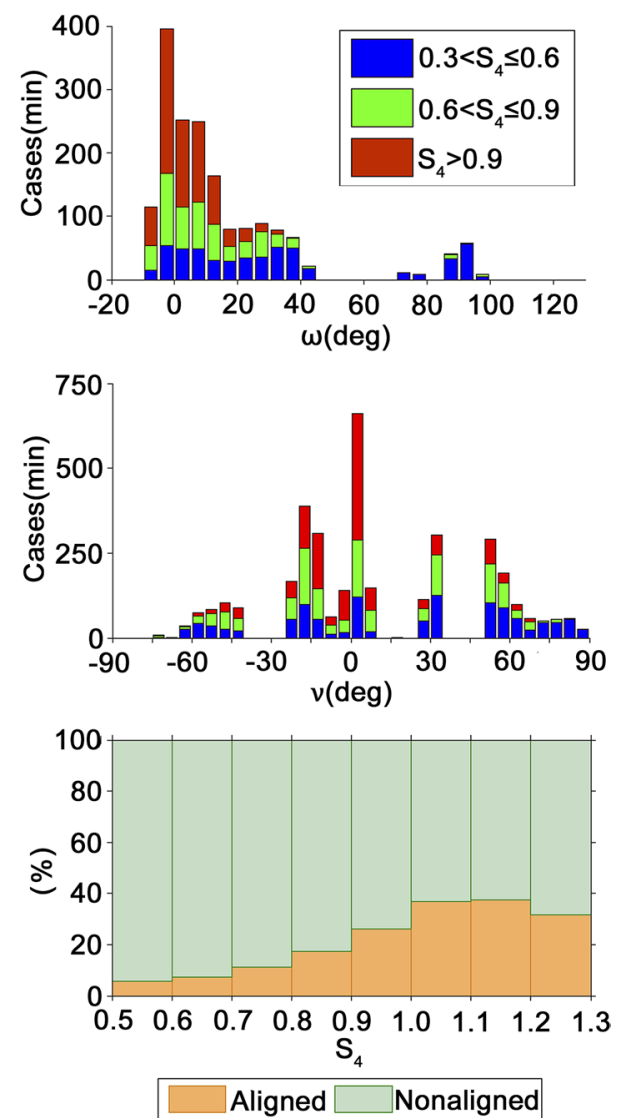

Fig. 5 Top and middle panels: number of L1 scintillation occurrences as a function of $\omega$ and $v$, respectively, for different ranges of $S_{4}$ values. Bottom panel: percentages of alignment and nonalignment as functions of $S_{4}$ intervals. Note that, for all $S_{4}$ intervals, the two percentages always add to $100 \%$
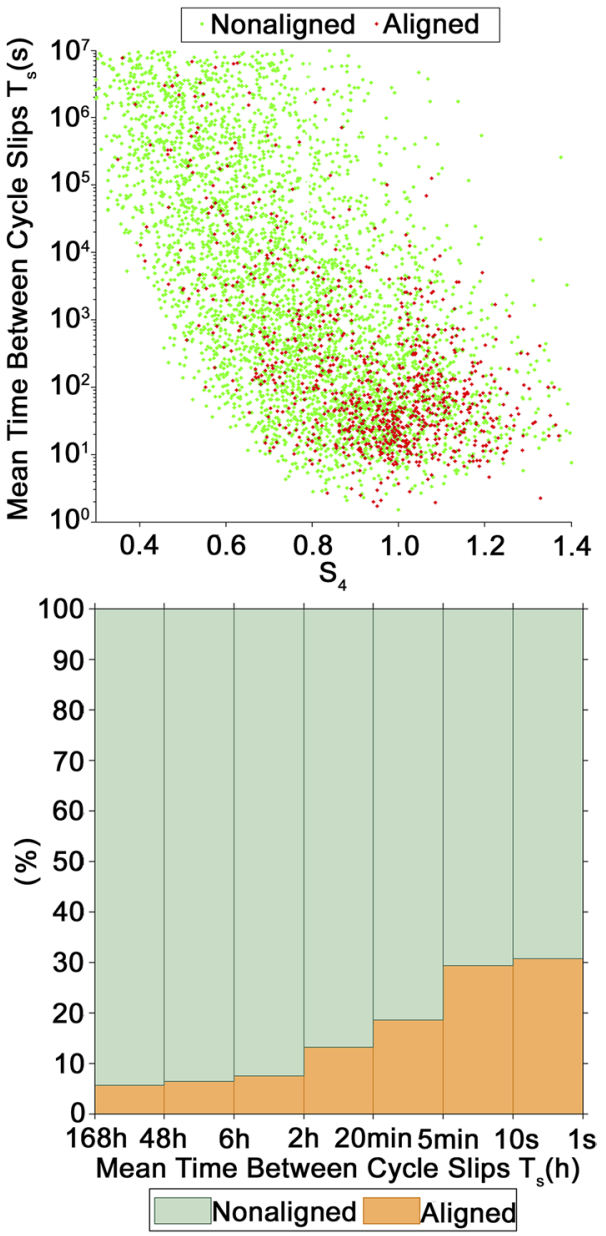

Fig. 6 Top panel: scatter plot of the mean time between cycle slips $T_{\mathrm{s}}$ as a function of $S_{4}$ for of aligned and nonaligned cases. Bottom panel: percentages of aligned and nonaligned cases for different $T_{\mathrm{s}}$ classes. Note that, for all $T_{\mathrm{s}}$ classes, the two percentages always add to $100 \%$

the aligned cases are concentrated in the region of high $S_{4}$ and low $T_{\mathrm{s}}$ values. The bottom panel in Fig. 6 shows the percentages of aligned and nonaligned cases for different $T_{\mathrm{s}}$ classes. Again, the $2 \%$ associated with each class add to $100 \%$. For very rare occurrences of cycle slips, $48 \mathrm{~h}<T_{\mathrm{S}}$ $\leq 168 \mathrm{~h}$, corresponding to one such event within the interval from 2 days to 1 week, the percentage of aligned cases is only $5.6 \%$. This percentage increases to $18.5 \%$ for $T_{\mathrm{S}}$ between 5 and $20 \mathrm{~min}$. However, for $1 \mathrm{~s}<T_{\mathrm{s}}<10 \mathrm{~s}$, the percentage of aligned cases reaches $30.7 \%$. Therefore, the alignment between the propagation path and the geomagnetic field directions favors the occurrence of the most serious conditions for the GPS receiver operation, leading to high values of $S_{4}$ and small values of $T_{\mathrm{S}}$.

Figure 7 displays the histograms for $\omega$ and $\nu$, conditioned by $T_{\mathrm{s}}<200 \mathrm{~s}$. They show the concentration of the most likely cases of loss of lock around $0^{\circ}$ for both angles $\omega$ and $\nu$. The percentages of $S_{4}$ classified by the alignment in Fig. 5 


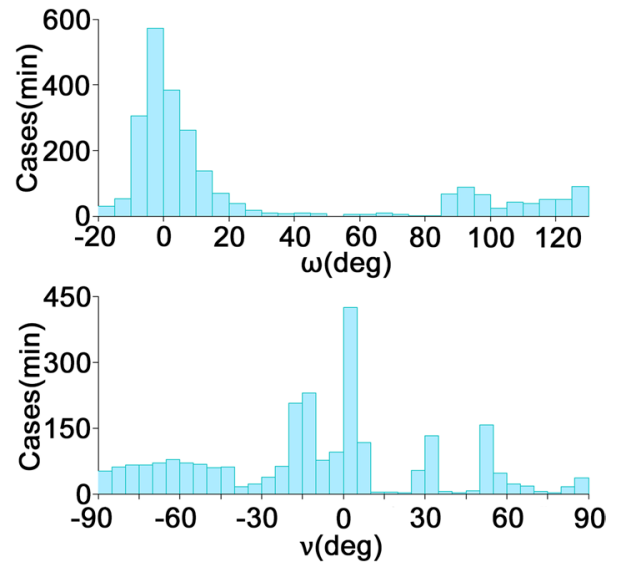

Fig. 7 Top panel: distribution of the angular difference between the path elevation and magnetic inclination for $T_{\mathrm{s}}<200 \mathrm{~s}$. Bottom panel: distribution of the value of the angular difference between the path azimuth and the geomagnetic declination for $T_{\mathrm{s}}<200 \mathrm{~s}$

and the present plots show that GPS signals received through field-aligned plasma bubbles may suffer enhancement in scintillation intensity. In view of the inverse relationship between $T_{\mathrm{s}}$ and $S_{4}$, evident in the top panel of Fig. 6, the distributions of the numbers of cases of $T_{\mathrm{s}}<200 \mathrm{~s}$ with respect to $\omega$ and $\nu$, which show their maximum occurrences at small angles, are consistent with top and middle panels of Fig. 5 . Such enhancement appears in addition to that occurring in the EIA crest region, arising from the fountain effect on the background plasma density. Scintillation enhancements due to the EIA crest region, northward of the GPS receiver, may also contribute to the alignment effect seen on the vertical plane; that is, as a function of $\omega$. However, the cases of enhanced scintillation intensity in which $T_{\mathrm{s}}<200 \mathrm{~s}$ for propagation alignment in azimuth $\nu$ do represent the scintillation enhancement for situations where the EIA crest effect is smaller. Both effects make signal tracking more difficult, with direct impact on the performance and availability of GPS receivers.
Table 1 presents the average and standard deviation values of $\alpha$, as well as its maximum recorded value for different $S_{4}$ intervals, considering aligned and nonaligned cases. It also presents the number of analyzed cases in each class. The analysis considers only the 1-min records affected by losses of lock, as indicated by the corresponding flag. It is observed that the average value of $\alpha$ for each aligned case is greater than the one for the corresponding nonaligned case. For all $S_{4}$ intervals, the increase of $\alpha$ values implies a decrease in the estimate of $T_{\mathrm{s}}$ as shown in Fig. 3. This is an indication that the alignment affects the statistics of amplitude scintillation in a severe way, with distributions that display a higher percentage of deep fades than those related with the nonaligned ones (Moraes et al. 2014b).

This section showed that GPS signals propagating along the magnetic field lines are more likely to propagate longer distances in the turbulent medium created by the fieldaligned EPBs. Therefore, the alignment geometry is more likely to cause interruptions to GPS receiver operation.

\section{Alignment and positioning}

This section discusses the influence of ionospheric irregularity alignments along the signal propagation paths on GPS positioning. The observation dataset was evaluated by the PPP approach (Zumberge et al. 1997) using the RT-PPP software developed by the Universidade Estadual Paulista Júlio de Mesquita Filho (UNESP), available at "http://is-cigal a-calibra.fct.unesp.br/ppp/" (Marques et al. 2014).

The PPP was applied with the standard configuration (that is, with corrections for tropospheric and first-order ionospheric refraction effects, but without any attempt to mitigate those from scintillation). Dual-frequency GPS data from the monitoring station were processed in the RTPPP with a sampling rate of $1 \mathrm{~s}$. The position parameters and receiver clock error were estimated at every epoch by the least-squares adjustment, while the phase ambiguities were estimated iteratively (Marques et al. 2016). Typically,
Table 1 Comparison between statistics of $\alpha$ coefficients for aligned and nonaligned conditions

\begin{tabular}{|c|c|c|c|c|c|c|c|c|}
\hline & \multicolumn{4}{|c|}{ Aligned } & \multicolumn{4}{|c|}{ Nonaligned } \\
\hline & $E(\alpha)$ & $\operatorname{Std}(\alpha)$ & $\operatorname{Max}(\alpha)$ & Cases & $E(\alpha)$ & $\operatorname{Std}(\alpha)$ & $\operatorname{Max}(\alpha)$ & Cases \\
\hline $0.3<S_{4} \leq 0.4$ & 3.16 & 4.37 & 51.20 & 42 & 2.58 & 2.59 & 22.93 & 2151 \\
\hline $0.4<\mathrm{S}_{4} \leq 0.5$ & 2.72 & 3.08 & 20.54 & 55 & 2.34 & 2.66 & 48.05 & 1549 \\
\hline $0.5<S_{4} \leq 0.6$ & 3.14 & 3.62 & 23.66 & 69 & 2.16 & 2.27 & 22.39 & 1176 \\
\hline $0.6<S_{4} \leq 0.7$ & 2.91 & 3.18 & 30.55 & 69 & 2.28 & 2.28 & 18.21 & 890 \\
\hline $0.7<S_{4} \leq 0.8$ & 2.83 & 2.70 & 22.16 & 91 & 2.21 & 1.67 & 13.74 & 733 \\
\hline $0.8<S_{4} \leq 0.9$ & 2.58 & 3.48 & 56.48 & 133 & 1.99 & 3.28 & 52.07 & 635 \\
\hline $0.9<S_{4} \leq 1.0$ & 2.01 & 1.09 & 12.38 & 188 & 1.82 & 3.13 & 43.86 & 534 \\
\hline $1.0<S_{4} \leq 1.1$ & 1.15 & 0.67 & 3.68 & 207 & 1.15 & 0.62 & 2.53 & 357 \\
\hline $1.1<S_{4} \leq 1.2$ & 1.02 & 0.48 & 2.20 & 100 & 0.90 & 0.44 & 1.68 & 166 \\
\hline $1.2<S_{4}$ & 0.81 & 0.37 & 1.62 & 57 & 0.65 & 0.34 & 1.40 & 123 \\
\hline
\end{tabular}


this procedure leads to a centimeter-level accuracy within $20 \mathrm{~min}$, due to the convergence period of the phase ambiguity parameters (Gao and Shen 2002). In the presence of scintillation, PPP may experience higher-order errors mainly associated with cycle slips and range degradations in the observables. Using the RT-PPP software, if a cycle slip is detected through the algorithm designed by Blewitt (1990), its corresponding ambiguity parameter is re-initialized. Consequently, a new convergence period is required for such parameter and the accuracy of the positioning may deteriorate. Another source of deterioration in positioning accuracy is the sudden change in geometry caused by losses of lock during scintillation, as highlighted by Zhang et al. (2014). The detection, identification and adaption (DIA) method (Teunissen 1998) is applied for outlier detection and quality control adjustment.

Typical examples of the influence of the alignment under discussion on the PPP performance is presented in Figs. 8 and 9, based on data recorded between 19:00 and 21:00 LT during the night of November 17, 2014. Figure 8 shows the skyplot of the time variation of the IPPs of all available GPS satellite links to the São José dos Campos monitor, in combination with the corresponding $S_{4}$ values. The satellite signals transmitted by PRN18, represented by G18 in the plot, are associated with ray paths aligned with magnetic-field elongated EPBs between 20:06 and 21:00 LT, being persistently and severely affected by the strongest scintillation. However, the alignment condition actually started earlier at 19:35 LT, with little noticeable effects.

The upper three panels of Fig. 9 present the total number of satellites used in the PPP solution, $S_{4}$ values for all

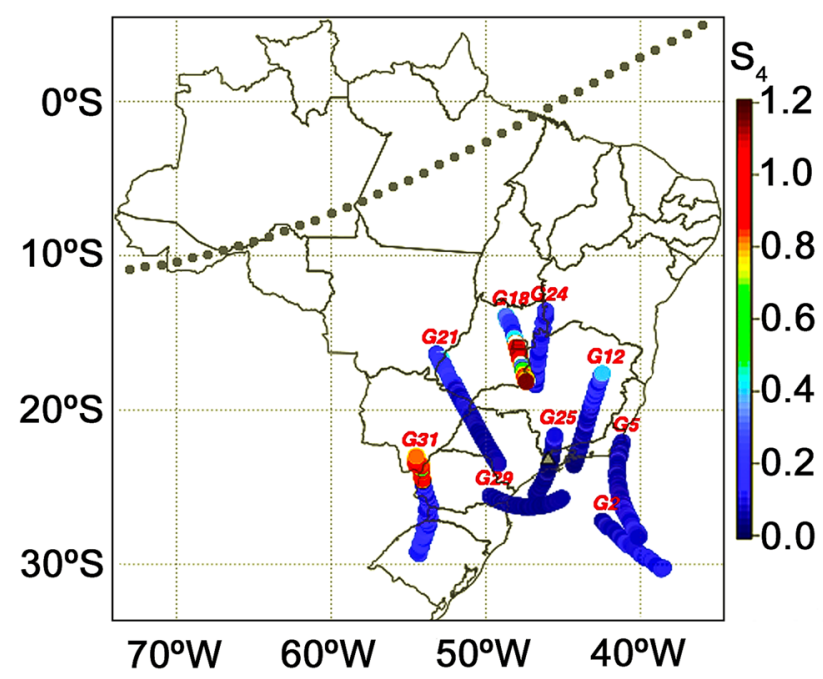

Fig. 8 Skyplot of the time variation of the IPPs of all available GPS satellite links to the São José dos Campos monitor in combination with the corresponding $S_{4}$ values for November 17, 2014 between 19:00 LT and 21:00 LT

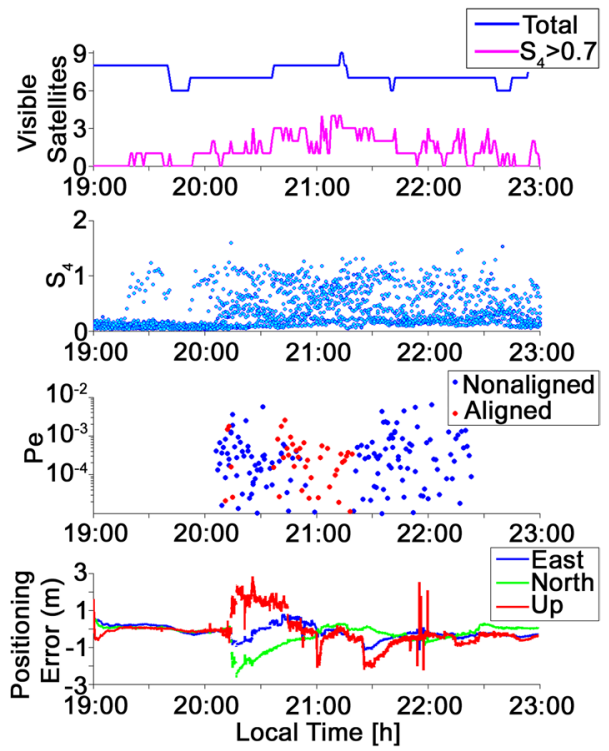

Fig. 9 Example from November 17, 2014. From top to bottom: total number of visible satellites used by the PPP processing approach; all $S_{4}$ values estimated between 19:00 and 23:00 LT; noticeable cases of bit error probability $P_{\mathrm{e}}>10^{-6}$ for aligned (red dots) and nonaligned cases (blue dots); and north (N), east (E) and up (U) components of the positioning error calculated by the PPP processing approach

satellites, and the noticeable cases of bit error probability $P_{\mathrm{e}}$ $>10^{-6}$, i.e., $T_{\mathrm{s}}<20000$ s. The bit error probability for the alignment condition, which only affected PRN18, started at approximately 20:06 LT and lasted until 21:00 LT, being indicated by red dots. However, the alignment precondition actually started at 19:35 LT, with lower $P_{\mathrm{e}}$ values. The bottom panel displays the time series of the north, east and up PPP error components. Analyzing the $S_{4}$ plot in Fig. 9, it is observed that scintillation with $S_{4}>0.7$ started at 19:40 LT, so remaining until 21:00 LT, while the PPP solution experienced higher-order errors, reaching, or sometimes exceeding, meter-level accuracy between 20:35 and 20:47 LT. These events occurred simultaneously with the period of EPB alignment with the PRN18 propagation path when noticeable values of $P_{\mathrm{e}}$ also occurred. This is evidence that $P_{\mathrm{e}}$ is a realistic parameter to diagnose difficulties in the PPP processing approach, not only under alignment, where it is more perceptible but also for $P_{\mathrm{e}}>10^{-6}$. What is quite relevant in the top panel is the fact that the transmissions by two satellites experienced simultaneous losses of lock during short intermittent intervals. These events are also identified by high $P_{\mathrm{e}}$ values, beginning at approximately 20:35 LT, according to the corresponding panel of Fig. 9. In the absence of adequate time for the PPP procedure to recover from losses of lock and converge, its solution yields large errors.

Table 2 presents detailed results from the highlighted night of November 17, 2014 (DOY 321). It also presents 
Table 2 PPP results under alignment conditions for November 15-30, 2014

\begin{tabular}{lllllll}
\hline DOY & Cases $S_{4}>0.7$ & $\operatorname{Max}\left(S_{4}\right)$ & $\operatorname{Max}\left(P_{\mathrm{e}}\right)$ & $\operatorname{RMS}(3 \mathrm{~d} \sigma)$ & $\operatorname{Max}(3 \mathrm{~d} \sigma)$ & $E(3 \mathrm{~d} \sigma)$ \\
\hline 321 & 30 & 1.28 & 0.0027 & 0.3623 & 2.0304 & 0.3770 \\
322 & 26 & 1.19 & 0.0047 & 0.4130 & 1.9380 & 0.5791 \\
323 & 48 & 1.37 & 0.0025 & 0.3241 & 2.5973 & 0.4232 \\
324 & 0 & 0.45 & 0.0000 & 0.1077 & 0.8250 & 0.3982 \\
325 & 51 & 1.60 & 0.0036 & 0.9226 & 2.8496 & 0.9686 \\
326 & 34 & 1.25 & 0.0030 & 0.3528 & 2.5637 & 0.6211 \\
327 & 43 & 1.22 & 0.0031 & 0.1888 & 1.2140 & 0.3040 \\
328 & 63 & 1.21 & 0.0035 & 0.1100 & 0.6216 & 0.3685 \\
329 & 52 & 1.41 & 0.0030 & 0.1020 & 0.6263 & 0.2716 \\
330 & 31 & 1.31 & 0.0012 & 0.1573 & 1.5378 & 0.5249 \\
331 & 46 & 1.33 & 0.0049 & 0.1996 & 1.2328 & 0.8322 \\
332 & 12 & 1.00 & 0.0014 & 0.2643 & 1.2503 & 0.7543 \\
333 & 8 & 1.06 & 0.0054 & 0.3266 & 2.3209 & 0.7251 \\
334 & 20 & 1.27 & 0.0013 & 0.4386 & 2.4038 & 1.2289 \\
\hline
\end{tabular}

observations for the period between November 18-30, 2014. All alignment cases occurred for PRN18. The three-dimensional RMS and average positioning error, calculated by the PPP processing approach, as well as its maximum value, all in meters, are listed in the last columns of the table, showing large PPP errors due to alignment. Such a result may be considered for mitigation of this type error in the future.

\section{Conclusion}

DasGupta et al. (2004) and Ray et al. (2014) previously demonstrated that GPS signals are more severely affected by scintillation when their propagation paths are aligned with EPBs. The former reference based their arguments on azimuth-elevation plots of the scintillation index estimated from measurements performed by a GPS monitor located at Calcutta, whose coordinates are $22.58^{\circ} \mathrm{N}, 88.38^{\circ} \mathrm{E}$ and dip latitude $17.35^{\circ} \mathrm{N}$, as well as on corresponding positioning errors, which reached $11 \mathrm{~m}$ in latitude and $8 \mathrm{~m}$ in longitude. The latter reference displayed $S_{4}$ values along GPS satellite tracks observed from Bhopal, whose coordinates are $23.28^{\circ} \mathrm{N}, 77.34^{\circ} \mathrm{E}$ and dip latitude $19.20^{\circ} \mathrm{N}$, in combination with a map of the angle between the ray path and the geomagnetic field directions, in terms of the subionospheric latitude and longitude. Noting that the example also represented observations from several other stations in the Indian sector, the authors also arrived at the above conclusion. Here, experimental data obtained during 32 days at a station located under the southern crest of the EIA in Brazil have been analyzed. The results have confirmed that, in the Brazilian sector, scintillation intensity is also enhanced when signal propagation path segments tend to align themselves with EPB structures, which are known to be elongated along magnetic flux tubes. Such scintillation enhancements occurred predominantly at azimuth angles around $345^{\circ}$, nearly aligned with the magnetic meridian over this region, which has a large westward declination $\left(21.4^{\circ} \mathrm{W}\right)$. Moreover, that loss of phase lock is more likely to occur in the presence of this geometry. The present situation favors the assessment of such effects. To extend the previous results, statistical analyses of the present experimental data have been performed. Next, the $\alpha-\mu$ model has been used to estimate the mean time between cycle slips $T_{\mathrm{s}}$ through its approximate relationship with the bit error rate $P_{\mathrm{e}}$ of binary DPSK signals. Thus, it has been quantitatively shown that the above alignment, which resulted in more severe scintillation cases with high values of $S_{4}$, is also responsible for small values of $T_{\mathrm{s}}$, likely causing losses of phase lock. The PPP analysis has shown that the large observed errors are related to that condition. The results have shown that PPP also experienced significant errors for high values of $P_{\mathrm{e}}$, even in the absence of the alignment condition. In one particular example, these errors increased in response to transmissions by two satellites experiencing simultaneous losses of lock during short intermittent intervals. These events strongly impacted GPS availability and positioning performance. However, it has been shown that such performance metrics have been especially degraded under the alignment condition.

Acknowledgements This work is supported by Conselho Nacional de Desenvolvimento Científico e Tecnológico $(\mathrm{CNPq})$ under award number (INCT) 465648/2014-2 and FAPESP 2017/50115-0. B. C. Vani thanks Federal Institute of Education, Science and Technology of Sao Paulo (IFSP) for supporting his Ph.D. research and Coordenação de Aperfeiçoamento de Pessoal de Nível Superior (CAPES) for Grant (CAPES/PDSE no. 19-2016/Process n. 88881.134266/2016-01). M. A. Abdu acknowledges the CAPES support for a senior visiting professorship at ITA/DCTA. E. Costa is supported by CNPq award number (PQ) 309013/2016-0. E. R. de Paula is supported by CNPq award number (PQ) 310802/2015-6. J. Sousasantos acknowledge CAPES for the financial support. The monitoring stations were deployed in 
the context of Projects CIGALA/CALIBRA, funded by the European Commission (EC) in the framework of FP7-GALILEO-2009-GSA and FP7-GALILEO-2011-GSA-1a, respectively, and FAPESP Project Number 06/04008-2. The authors thank the reviewers for the insightful and constructive comments, which helped them in the development of a better paper.

\section{References}

Abdu MA, Bittencourt JA, Batista IS (1981) Magnetic declination control of the equatorial $\mathrm{F}$ region dynamo electric field development and spread F. J Geophys Res 86(13):11443-11446. https://doi. org/10.1029/JA086iA13p11443

Abdu MA, Kherani EA, Batista IS, de Paula ER, Fritts DC, Sobral JHA (2009) Gravity wave initiation of equatorial spread F/ plasma bubble irregularities based on observational data from the SpreadFEx campaign. Ann Geophys 27(7):2607-2622. https ://doi.org/10.5194/angeo-27-2607-2009

Abdu MA, Brum CGM, Batista PP, Gururbaran S, Pancheva D, Bageston JV, Batista IS, Takahashi H (2015) Fast and ultrafast Kelvin wave modulations of the equatorial evening $F$ region vertical drift and spread F development. Earth Planets Space 67(1):1-15. https ://doi.org/10.1186/s40623-014-0143-5

Basu S, Basu S (1981) Equatorial scintillations-a review. J Atmos Terr Phys 43(5-6):473-489. https://doi.org/10.1016/00219169(81)90110-0

Blewitt G (1990) An automatic editing algorithm for GPS data. Geophys Res Lett 17(3):199-202. https://doi.org/10.1029/GL017 i003p00199

DasGupta A, Ray S, Paul A, Banerjee P, Bose A (2004) Errors in position-fixing by GPS in an environment of strong equatorial scintillations in the Indian zone. Radio Sci, 39(1), https://doi. org/10.1029/2002RS002822

de Paula ER, Jonah OF, Moraes AO, Kherani EA, Fejer BG, Abdu MA, Muella MTAH, Batista IS, Dutra SLG, Paes RR (2015) Low-latitude scintillation weakening during sudden stratospheric warming events. J Geophys Res 120(3):2212-2221. https://doi. org/10.1002/2014JA020731

Doherty PH, Delay SH, Valladares CE, Klobuchar JA (2004) Ionospheric scintillation effects on GPS in the equatorial and auroral regions. J Inst of Navig 50(4):235-246. https://doi. org/10.1002/j.2161-4296.2003.tb00332.x

El Ayadi MMH, Ismail MH (2014) Novel closed-form exact expressions and asymptotic analysis for the symbol error rate of single- and multiple-branch MRC and EGC receivers over $\alpha-\mu$ fading. IEEE Trans Veh Technol 63(9):4277-4291. https://doi. org/10.1109/TVT.2014.2316418

Gao Y, Shen X (2002) A new method for carrier-phase-based precise point positioning. J Inst Navig 49(2):109-116. https://doi. org/10.1002/j.2161-4296.2002.tb00260.x

Humphreys TE, Psiaki ML, Hinks JC, O'Hanlon B, Kintner PM (2009) Simulating ionosphere-induced scintillation for testing GPS receiver phase tracking loops. IEEE J Sel Top Signal Process 3(4):707-715. https://doi.org/10.1109/JSTSP.2009.2024130

Humphreys TE, Psiaki ML, Ledvina BM, Cerruti AP, Kintner PM (2010a) Data-driven testbed for evaluating GPS carrier tracking loops in ionospheric scintillation. IEEE Trans Aerosp Electron Syst 46(4):1609-1623. https://doi.org/10.1109/TAES.2010.55955 82

Humphreys TE, Psiaki ML, Kintner PM Jr (2010b) Modeling the effects of ionospheric scintillation on GPS carrier phase tracking. IEEE Trans Aerosp Electron Syst 46(4):1624-1637. https:// doi.org/10.1109/TAES.2010.5595583
Kintner PM, Kil H, Beach TL, de Paula ER (2001) Fading timescales associated with GPS signals and potential consequences. Radio Sci 36(4):731-743. https://doi.org/10.1029/1999RS002310

Kintner PM, Ledvina BM, de Paula ER (2007) GPS and ionospheric scintillations. Space Weather 5(9):1-23. https://doi. org/10.1029/2006SW000260

Magableh AM, Matalgah MM (2009) Moment generating function of the generalized $\alpha-\mu$ distribution with applications. IEEE Commun Lett 13(6):411-413. https://doi.org/10.1109/LCOMM.2009.09033

Marques HA, Monico JFG, Shimabukuro MH, Oyama RT, Wentz JP (2014) Real time PPP: fundamentals, computational implementation and results analysis for static and kinematic mode. Braz Cartogr Mag 6(66):1331-1345

Marques HAS, Monico JFG, Marques HA (2016) Performance of the L2C civil GPS signal under various ionospheric scintillation effects. GPS Solut 20(2):139-149. https://doi.org/10.1007/s1029 1-015-0472-2

Moraes AO, de Paula ER, Perrella WJ, Rodrigues FS (2012) On the distribution of GPS signal amplitudes during the low-latitude ionospheric scintillation. GPS Solut 17(4):499-510. https://doi. org/10.1007/s10291-012-0295-3

Moraes AO, de Paula ER, Muella MTAH, Perrella WJ (2014a) On the second order statistics for GPS ionospheric scintillation modeling. Radio Sci 49(2):94-105. https://doi.org/10.1002/2013RS005270

Moraes AO, Costa E, de Paula ER, Perrella WJ, Monico JFG (2014b) Extended ionospheric amplitude scintillation model for GPS receivers. Radio Sci 49(5):315-333. https://doi. org/10.1002/2013RS005307

Moraes AO, Costa E, Abdu MA, Rodrigues FS, de Paula ER, Oliveira K, Perrella WJ (2017) The variability of low-latitude ionospheric amplitude and phase scintillation detected by a triplefrequency GPS receiver. Radio Sci 52(4):439-460. https://doi. org/10.1002/2016RS006165

Moreno B, Radicella S, de Lacy MC, Herraiz M, Rodriguez-Caderot G (2011) On the effects of the ionospheric disturbances on precise point positioning at equatorial latitudes. GPS Solut 15(4):381390. https://doi.org/10.1007/s10291-010-0197-1

Ray S, Bhowmik U, Das A (2014) Scintillation effects related to propagation geometry as applicable to Indian SBAS. In: Proceedings of the XXXI URSI General Assembly and Scientific Symposium, paper 1463. Beijing

Sobral JHA, Abdu MA, Takahashi H, Taylor MJ, de Paula ER, Zamlutti CJ, Aquino MG, Borba GL (2002) Ionospheric plasma bubble climatology over Brazil based on 22 years (1977-1998) of 630 nm airglow observations. J Atmos Sol Terr Phys 64(12-14):15171524. https://doi.org/10.1016/S1364-6826(02)00089-5

Teunissen PJG (1998) Quality control and GPS. In: Teunissen PJG, Kleusberg A (eds) GPS for geodesy, 2nd edn. Springer, Berlin, pp 271-318

Thébault $\mathrm{E}$ et al (2015) International geomagnetic reference field: the 12th generation. Earth Planets Space 67:1-19. https://doi. org/10.1186/s40623-015-0228-9. 79

Tsunoda RT (1985) Control of the seasonal and longitudinal occurrence of equatorial scintillations by the longitudinal gradient in integrated $\mathrm{E}$ region PedersEn conductivity. J Geophys Res 90(A1):447-456. https://doi.org/10.1029/JA090iA01p00447

Vani BC, Shimabukuro MH, Monico JFG (2016) Visual exploration and analysis of ionospheric scintillation monitoring data: the ISMR query tool. Comp Geosc 104:125-134. https://doi. org/10.1016/j.cageo.2016.08.022

Xu R, Liu Z, Li M, Morton Y, Chen W (2012) An analysis of lowlatitude ionospheric scintillation and its effects on precise point positioning. J Global Position Syst 11(1):22-32. https://doi. org/10.5081/jgps.11.1.22 
Yacoub MD (2007) The $\alpha-\mu$ distribution: A physical fading model for the Stacy distribution. IEEE Trans Veh Technol 56(1):27-34. https://doi.org/10.1109/TVT.2006.883753

Yeh KC, Liu CH (1982) Radio wave scintillations in the ionosphere. Proceedings of the IEEE, 70(4):324-360, https://doi.org/10.1109/ PROC.1982.12313

Zhang X, Guo F, Zhou P (2014) Improved precise point positioning in the presence of ionospheric scintillation. GPS Solut 18(1):51-60. https://doi.org/10.1007/s10291-012-0309-1

Zumberge JF, Heflin MB, Jefferson DC, Watkins MM, Webb FH (1997) Precise point positioning for the efficient and robust analysis of GPS data from large networks. J Geophys Res 102(B3):50055017. https://doi.org/10.1029/96JB03860

Alison de O. Moraes received the B.S. in telecommunications engineering from Universidade de Taubaté, UNITAU, Brazil in 2003, and Dr.Sc degree in the School of Electronic and Computer Engineering at Instituto Tecnológico de Aeronáutica, ITA, Brazil in 2013. Engineer at the Instituto de Aeronáutica e Espaço IAE since 2004, and graduate professor at ITA since 2015.

Bruno C. Vani received the B.S. in Computer Science (2011) and M.Sc. (2014) in Cartographic Sciences from Univ. Estadual Paulista (UNESP). Currently, he is a Ph.D. candidate at UNESP. Since 2014, he is a lecturer at the Instituto Federal de São Paulo (IFSP/SP), Presidente Epitácio, Brazil.

Emanoel Costa received the BS and MS degrees in electrical engineering from Instituto Militar de Engenharia, Brazil in 1972 and 1974 and the Ph.D. degree from Cornell University, USA, in 1978. He has been with CETUC/PUC-Rio, Brazil, since 1978, with research interests in the areas of tropospheric and ionospheric effects on radio wave propagation.

Mangalathayil A. Abdu received Ph.D. degree, in 1967, from Gujarat University India. He has been a postdoctoral research fellow at the University of Western Ontario (1968-71), a professor at CRAAM Mackenzie University, Sao Paulo (1971-73), research scientist since 1973 at the National Institute for Space Research (INPE), and presently a visiting professor at ITA. His research interests are in space weather and ionospheric physics.
Eurico R. de Paula attended the M.Sc. degree at Telecommunications at ITA, and Dr.Sc. in Geophysics at INPE, where he has been working as a Researcher for 40 years. He attended pos-doc programs at USU, Utah State University, Logan, USA for 5 years. His main area of interest is ionospheric physics with a focus on ionospheric scintillations detected by radars and GPS receivers.

Jonas Sousasantos graduated in Mathematics at the Universidade de Taubaté, UNITAU, Brazil in 2010. Dr.Sc. (2017) and M.Sc. (2013) degrees obtained at the National Institute for Space Research. He is currently a Postdoctoral Associate at Instituto Tecnológico de Aeronáutica, ITA. His main area of interest is mathematical modeling and forecasting of ionospheric irregularities and atmospheric-ionospheric vertical coupling.

João F. G. Monico graduated in Cartographic Eng. from UNESP (1982), Master in Geodetic Science from UFPR (1988) and Ph.D. on Space Geodesy from Nottingham University (1995). Has experience in Geosciences, focusing on the following subjects: GNSS for Geodesy and Atmosphere, Quality Control on Geodesy. Dr. Galera Monico was indicated as one of the 50 GNSS Leaders to Watch by the GPS World magazine.

Biagio Forte graduated in Physics at the University of Trieste (Italy) and went on to complete Ph.D. in Geo-Physics at the Karl-Franzens University of Graz (Austria). Biagio joined the Department of Electronic and Electrical Engineering in 2012 as a Prize Fellow in Space Weather.

Patrícia Mara de Siqueira Negreti graduated in Physics from Universidade Estadual Paulista Júlio de Mesquita Filho (2003), where he received four awards/honors. Masters in Medical Physics by the Institute of Energy and Nuclear Research (2006) and Ph.D. in Space Geophysics by the National Institute of Space Research (2012).

Milton Hirokazu Shimabukuro Assistant Professor at UNESP, Presidente Prudente, São Paulo, Brazil, since 2006. He received B.S. degree in Computer Science and a doctorate in Computer Science and Computational Mathematics, both from Institute of Mathematics and Computer Sciences, University of São Paulo, Brazil. His research interests are visual analytics, organization, and visualization of spatial and temporal data. 